\title{
Magnetically enhanced vacuum arc thruster
}

\author{
Michael Keidar ${ }^{1}$, Jochen Schein ${ }^{2,4}$, Kristi Wilson ${ }^{2}$, \\ Andrew Gerhan ${ }^{2}$, Michael Au², Benjamin Tang ${ }^{2}$, \\ Luke Idzkowski $^{2}$, Mahadevan Krishnan ${ }^{2}$ and Isak I Beilis ${ }^{3}$ \\ ${ }^{1}$ University of Michigan, Ann Arbor 48109 MI, USA \\ 2 Alameda Applied Science Corporation, San Leandro, CA 94577, USA \\ ${ }^{3}$ Tel Aviv University, Tel Aviv, Israel \\ E-mail: keidar@umich.edu
}

Received 24 May 2005, in final form 9 August 2005

Published 12 September 2005

Online at stacks.iop.org/PSST/14/661

\begin{abstract}
A hydrodynamic model of the vacuum arc thruster and its plume is described. Primarily an effect of the magnetic field on the plume expansion and plasma generation is considered. Two particular examples are investigated, namely the magnetically enhanced co-axial vacuum arc thruster (MVAT) and the vacuum arc thruster with ring electrodes (RVAT). It is found that the magnetic field significantly decreases the plasma plume radial expansion under typical conditions. Predicted plasma density profiles in the plume of the MVAT are compared with experimental profiles, and generally a good agreement is found. In the case of the RVAT the influence of the magnetic field leads to plasma jet deceleration, which explains the non-monotonic dependence of the ion current density, on an axial magnetic field observed experimentally.

(Some figures in this article are in colour only in the electronic version)
\end{abstract}

\section{Introduction}

In the late 1960s and early 1970s several experimental groups investigated the use of vacuum arcs in electric thrusters for spacecraft propulsion (see review papers $[1,2]$ and references therein). The main reason for considering vacuum arcs for electrical propulsion was the nature of the plasma plume expanding from the cathode, which exhibits a high velocity and is highly directional. In general a vacuum arc is established by using a coaxial geometry with a relatively small diameter cathode which is surrounded by an insulator and an anode. Vacuum arcs can be produced from any electrically conductive material. The suitability of various materials for vacuum arc propulsion was first studied by Dethlefsen [3]. In the case of a magnesium cathode, a plasma velocity of $50000 \mathrm{~m} \mathrm{~s}^{-1}$ was demonstrated, which is certainly very appealing to the propulsion community. Pulsed operation was demonstrated and the thrust efficiency was found to be dependent on the electrode material, the amplitude and duration of the current pulse.

\footnotetext{
4 Now with Lawrence Livermore Nat. Lab., Livermore, CA, USA.
}

One reason for the material dependence is the fact that metals with a low melting point and low thermal conductivity erode rather heavily, with a large part of the erosion resulting in macrodroplets. These droplets tend to reduce the thrust efficiency because they are not accelerated to as high velocities as are the ions. While this reduces thrust efficiency, it has to be pointed out that the droplet emission does not depend on material properties alone and hence can be minimized through the action of thermal and mechanical inertia if a current pulse of sufficiently short duration on the order of $10 \mu \mathrm{s}$ is used in a pulsed vacuum arc thruster (VAT).

Apart from the high exit velocity and the fact that the cathode material can be chosen from a large variety of candidates, pulsed VATs were found to have other appealing characteristics, which include the use of a solid fuel, low voltage operation, as the burning voltage of vacuum arcs does not exceed $100 \mathrm{~V}$, and simple control by adjusting the arc current and repetition rate.

Very recently, interest in the vacuum arc source for propulsion applications was renewed. The first use employed the vacuum arc source as a plasma source for the ion thruster [4]. The measured energy efficiency was about $80 \%$ 
that is comparable to that of the Xenon ion thruster. The development of the vacuum arc source for an ion thruster led to the design of a thruster based on the pulsed vacuum arc without any ion extraction [5]. For latter configuration, a specific impulse of about $1000 \mathrm{~s}$ was measured and $21 \%$ of efficiency was estimated. Further development of this technology is attributed primarily to Alameda Applied Science Corporation (AASC).

\subsection{AASC vacuum arc thruster}

The VAT is a simple, yet efficient electric propulsion device that combined with an inductive energy storage power processing unit (PPU) results in a low mass $(<300 \mathrm{~g})$ system. The basic mechanism underlying the VAT is the production of micrometre-size fully ionized micro-plasmas (cathode spots) on the cathode surface, which expand into vacuum at high velocities, with the ions producing thrust. Every cathode spot carries a limited amount of current $(\sim 1-10 \mathrm{~A})$ and exists for a few nanoseconds, which makes the VAT very scalable with respect to pulsed operation and/or amount of plasma produced.

The need for a low mass propulsion system motivated the development of the VAT system. A pulsed plasma thruster that uses conductive cathode materials as the propellant is combined with an energy storage power processing unit that takes $5-24 \mathrm{~V}$ from the bus and converts it into an adequate power pulse for the thruster. It is a system well suited to providing small impulse bits $(\approx 1 \mu \mathrm{Ns})$ at high specific impulse, $I_{\mathrm{sp}}$ of about 1000-3000s. Applications include positioning and drag makeup for small, power and mass limited, satellites.

The performance of the VAT is determined by the propellant mass, the degree of ionization of the plasma, the angle of expansion, the average charge state and the ion velocity. All these parameters have been measured repeatedly in the past and verified for numerous materials and operating conditions [5-8]. Typical values for the ion velocity vary between 10 and $30 \mathrm{~km} \mathrm{~s}^{-1}$, the average arc/ion current ratio has been shown to be of the order of $8 \%$ and a cosine distribution has been found to emulate the plasma plume expansion very well.

In order to produce a low-mass system, the VAT was constructed using an inductive energy storage (IES) circuit PPU and simple thruster head geometry. In the PPU, an inductor is charged through a semiconductor switch. When the switch is opened, a voltage peak, $L \mathrm{~d} I / \mathrm{d} t$, is produced, which breaks down the thin metal film coated anode cathode insulator surface at relatively low voltage levels $(\approx 200 \mathrm{~V})$. The current that was flowing in the solid-state switch (for $\leqslant 1 \mathrm{~ms}$ ) is fully switched to the vacuum arc load. Typical currents of $\sim 100 \mathrm{~A}$ (for $\sim 100-500 \mu \mathrm{s}$ ) are conducted with voltages of $\sim 25-30 \mathrm{~V}$. Consequently, most of the magnetic energy stored in the inductor is deposited into the plasma pulse. The efficiency of the PPU may thus be $>90 \%$. By varying the length of the trigger signal, the level of the current in the switch and thereby the energy stored in the inductor can also be adjusted. This in turn changes the amount of energy transferred to the arc and the impulse bit of the individual pulse. Obviously, the repetition rate of the individual pulse can be changed by varying the input signal as well. (a)

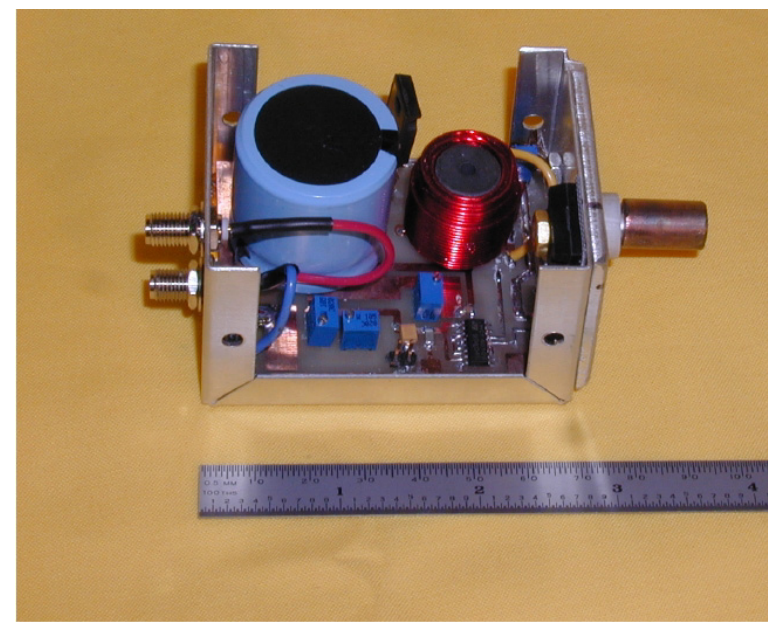

(b) $\mathrm{L}$

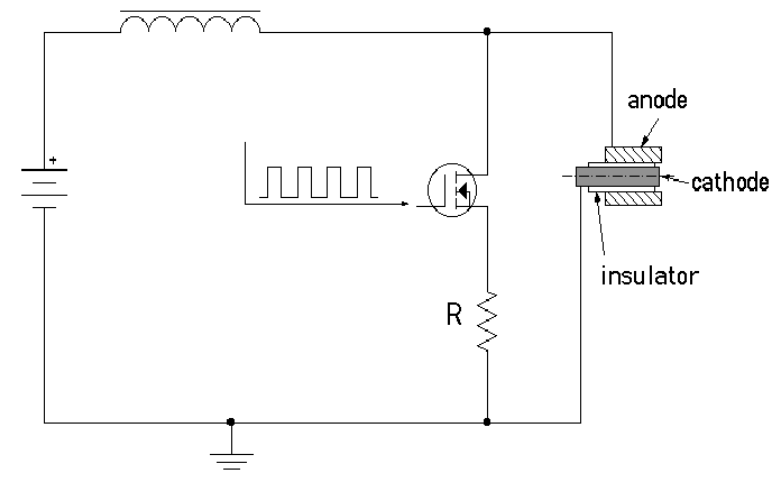

Figure 1. (a) VAT system including PPU (thruster head is on the right). (b) VAT equivalent circuit.

The mass of the PPU is small $(<300 \mathrm{~g})$, resulting in a low-mass system. The plasma output is quasi-neutral, and therefore no additional neutralizer is needed. An EMI filter might be necessary due to the noisy characteristics of the discharge, high peak currents and fast switching. A drawback for this technology is possible contamination from the metal propellant. A low-mass feed mechanism is available, and therefore even long missions can use this technology.

A picture of the VAT system, along with an equivalent circuit is, shown in figures $1(a)$ and $(b)$. Additional information about the principle of the VAT can be found elsewhere [6-8].

While the system is simple and can be developed with an extremely low mass, the divergence of the plasma plume reduces the efficiency of the system due to the variations in the thrust vector and also increases the chances of contamination of essential parts of the spacecraft. Based on prior work by Gilmour and co-workers $[9,10]$, who achieved a total efficiency of $30 \%$ with a copper cathode and a magnetic field of $500 \mathrm{G}$ by using a coaxial arc-diode configuration with an additional magnetic coil because a magnetic field can be used to direct a vacuum arc plasma [11,36] an axial magnetic field was used to control the plasma flow. The magnetic field is produced by a coil wrapped around the anode of a coaxial thruster head (figure 2). For the purpose of performing initial experiments, 
(a)

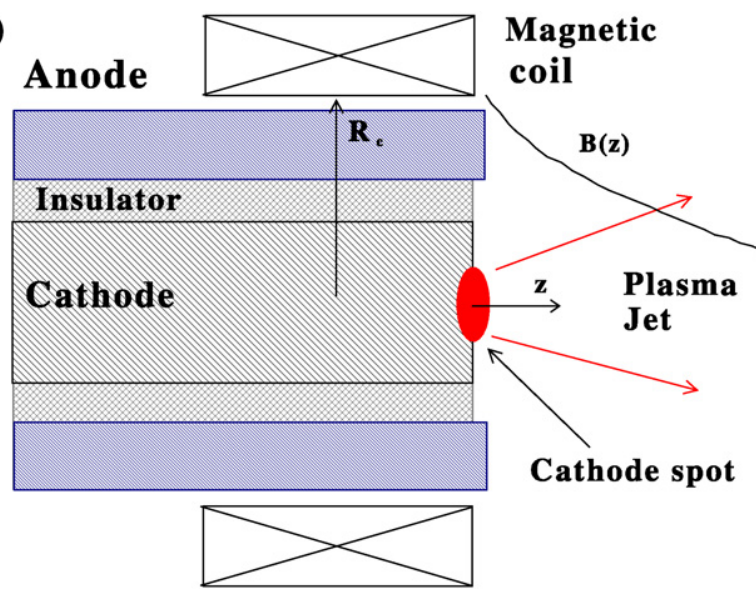

(b)

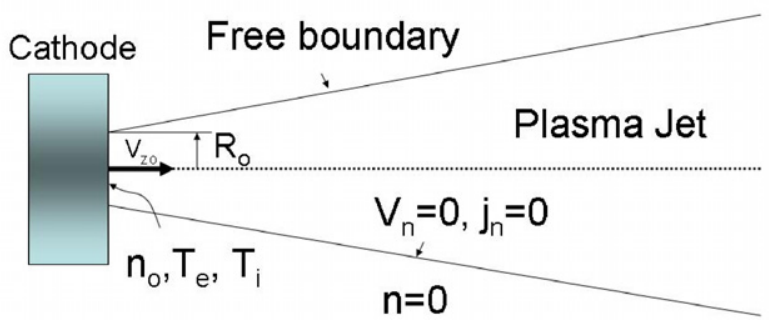

Figure 2. Schematic drawing of the MVAT and computational domain.

the currents in the coil and in the thruster are controlled independently, while a final design will utilize the magnetic field produced in the inductor of the IES PPU to control the plasma plume [12].

It has been found that by applying an axial magnetic field $(0.1 \mathrm{~T})$ around the cathode not only the plasma plume is confined but also that the ion velocities increase, leading to an improved thrust-to-power ratio. The basic vacuum arc properties that are essential for understanding VAT operation will be analysed below.

\subsection{Vacuum arc properties}

The metal plasma formed by a vacuum arc is created on the cathode surface at cathode spots. Optical observations of various resolutions indicate that the spot has a homogeneous bright region or consists of cells and fragments with a typical total size of about 10-100 $\mu \mathrm{m}$ [13-15]. The cathodic plasma expands away from the cathode in a direction normal to the surface with a speed that is typically $10-30 \mathrm{~km} \mathrm{~s}^{-1}$. This magnitude is as much as ten times the speed of sound [16]. The pioneering work of Plyutto et al [17] and Davis and Miller [18] demonstrated that more than $95 \%$ of the material that evaporates from the spot surface emerges from the spot plasma as ions. The characteristics of vacuum arc plasmas have been investigated and described by many authors $[15,19,20]$. An interesting feature of the vacuum arc, in contrast to a gaseous plasma, is that the vacuum arc plasma is fully ionized and has a mean charge state generally greater than unity. The angular ion current density distribution and the radial profile of the ion current and plasma potential were studied [21,22]. The measured ion distribution without a magnetic field was a slightly flattened cosinusoidal function. With an axial magnetic field, the ion distribution became peaked along the axial axis. Recently, the ion charge state distribution [23,24], and ion velocities [25-27] were measured using time-of-flight and current 'spike' techniques. These measurements were performed for most of the conducting elements of the periodic table, and a correlation between the element dependent ion velocity and average charge state was found. Refractory metal ions such as Mo and $\mathrm{W}$ have the highest energy $(150-200 \mathrm{eV})$. This energy is much higher than the arc voltage equivalent (about $20 \mathrm{~V}$ ), therefore leaving open the question of the ion acceleration mechanism in the cathode spot. Theoretically it was shown that the gas-dynamic mechanism is responsible for ion acceleration $[28,29]$. A more complete self-consistent model of the plasma acceleration in the cathode spot jet was developed by Beilis and co-workers $[30,31]$. They concluded that the supersonic velocity of the cathode plasma jet is due to momentum transfer from the electrons to the ions by friction and an ambipolar electric field. A high-pressure plasma in the cathode spot creates the reaction force on the cathode (thrust) as schematically shown in figure 2 . In the 1930s it was found experimentally that the plasma stream exerts an anomalously large reaction force on the cathode, which is about $17 \pm 3 \mathrm{dyn} \mathrm{A}^{-1}$ (or $\sim 0.2 \mathrm{mN} \mathrm{A}^{-1}$ ) [32-35]. It is very important to note that since the reaction force is proportional to the arc current, the thrust can be controlled easily.

\subsection{Vacuum arc plasma jet modelling}

In the vacuum arc, the plasma jet expansion strongly depends on the processes occurring in the near cathode region in which plasma is generated. In addition, the character of the plasma expansion depends on the inter-electrode gap geometry. In principle the problem of the plasma expansion in the vacuum arc has two-dimensional features. The first consistent two-dimensional analysis of the plasma flow taking into account both plasma generation effects and geometry was performed by Keidar and co-workers [36-40]. In these models the effect of an externally imposed magnetic field was also studied. The peculiarity of the proposed approach is in the fact that the plasma boundary (free boundary) was treated selfconsistently. It was found that a plasma jet has a conical shape and imposition of an axial magnetic field significantly alters plasma expansion. The magnetic force from an azimuthal selfmagnetic field was also taken into account. It was found that the self-magnetic field does not affect the plasma jet shape, density, velocity and current distribution for arc currents less than $200 \mathrm{~A}$. On the other hand, the mass and the current flow are compressed in a high current vacuum arc that indicates the significance of the self-magnetic field. While studying the geometry effect in combination with an axial magnetic field it was found that the near anode region plays an important role. For instance, in the case of a ring anode vacuum arc, the plasma voltage and anode voltage contribute substantially to the total arc voltage. The arc voltage increases with imposition of a magnetic field and decreases with the anode length in agreement with experiment. Multiply charged ion transport in the free boundary vacuum arc plasma jet was studied [41,42]. 
It was also found that the differently charged ions of the same element have approximately the same velocity. Keidar and co-workers $[41,42]$ found that the charge state distribution is not non-uniform along the plasma jet due to the separation of different charge species. Due to the existence of an electric field in the quasi-neutral plasma the different charged ion species can be separated according to this model. It was found that the mean charge state distribution is strongly non-uniform with a tendency for a highly charged species to appear near the plasma jet boundary region. This prediction was confirmed by experiments [42]. The above-mentioned models relied on the assumption that the plasma expansion is isothermal. However, recent complementary analysis of the two-dimensional energy balance indicated that even in the high-current vacuum arc the temperature changes are marginal [43].

In order to understand the influence of the magnetic field and to provide a recommendation for a highly efficient thruster design, we develop a model of the magnetically enhanced vacuum arc thruster (MVAT). The model is based on the previously developed hydrodynamic formulation of the cathodic plasma jet expansion problem and takes into account peculiarities of the plasma flow in two MVAT configurations. In particular in the case of the co-axial VAT we take into account magnetic field weakening in the plasma plume region, while in the case of the ring anode VAT we consider cathodic plasma jet flow across the magnetic field. The rest of this paper is organized as follows. In section 2 the model of the VAT is described. In section 3 we describe the application of the model for a MVAT. This is followed by an analysis of the ring-electrode VAT configuration. In section 4 we summarize our results and discuss the most important findings.

\section{The hydrodynamic model of the vacuum arc thruster}

According to the vacuum arc cathode jet model the plasma has a density of about $10^{20}-10^{21} \mathrm{~m}^{-3}$ in the inter-electrode gap at about $1 \mathrm{~mm}$ from the cathode plane [31]. In such a density range the influence of inelastic collisions (ionization, recombination, etc) on the plasma density can be neglected. The plasma jet has a free radial boundary whose position will be determined as part of a self-consistent solution. The plasma jet model is based on the following assumptions and conditions: (1) the vacuum arc plasma is fully ionized; (2) during the expansion, the temperature of each species remains constant; (3) the mean free path for elastic electron-ion collisions is much smaller than the characteristic plasma jet radius; (4) the ions and electrons are ideal gases with partial pressures $P_{\alpha}=k T_{\alpha} N_{\alpha}$, where $\alpha=\mathrm{e}$, i and $k$ is Boltzmann's constant; (5) the electrons are magnetized and the ions are unmagnetized, i.e. $\rho_{\mathrm{i}} \gg R_{0} \gg \rho_{\mathrm{e}}$, where $\rho_{\mathrm{e}}, \rho_{\mathrm{i}}$ are the electron and ion Larmor radii, respectively; (2) the plasma is quasi-neutral.

Briefly, the model is based on two-dimensional, axisymmetric, two-fluid magnetohydrodynamics that includes mass and momentum conservation for both the ions and electrons [36-40]. The following system of equations completely describes the plasma flow and currents distribution:

$$
m_{\mathrm{i}}\left(\boldsymbol{V}_{\mathrm{i}} \cdot \nabla\right) \boldsymbol{V}_{\mathrm{i}}=-k\left(Z_{\mathrm{i}} T_{\mathrm{e}}+T_{\mathrm{i}}\right) \cdot \nabla \ln (n)+\boldsymbol{j} \times \boldsymbol{B} / n,
$$

$$
\begin{gathered}
\boldsymbol{j}=\sigma\left\{\boldsymbol{E}+\left(k T_{\mathrm{e}} / e\right) \cdot \boldsymbol{\nabla} \ln (n)-\boldsymbol{j} \times \boldsymbol{B} /(e n)+\left(\boldsymbol{V}_{\mathrm{i}} \times \boldsymbol{B}\right)\right\}, \\
\nabla \cdot\left(\boldsymbol{V}_{\mathrm{i}} n\right)=0, \\
\boldsymbol{\nabla} \cdot \boldsymbol{j}=0,
\end{gathered}
$$

where $m_{\mathrm{i}}$ is the ion mass, $V_{\mathrm{i}}$ is the ion velocity, $T_{\mathrm{e}}$ and $T_{\mathrm{i}}$ are electron and ion temperatures, $n$ is the plasma density, $\boldsymbol{E}$ is the electric field and $\boldsymbol{j}$ is the current density. This model assumes that during jet expansion the temperature of each species does not change substantially. Recent calculations of the electron temperature variations in the inter-contact gap of the vacuum arc support this assumption [43]. The plasma flow is determined by the boundary conditions at the cathode. The plasma is simulated until it leaves the computational domain which is extended in the axial direction up to $50 \mathrm{~mm}$.

There are three boundaries where conditions must be specified: the cathode side, the anode side and the free lateral boundary as shown in figure $2(b)$. The formulation of the boundary conditions was described elsewhere [36,37]. Here we just briefly overview the boundary conditions that determine the physical relevance of the solution. On the cathode side, we choose for the starting plane of the present two-dimensional model some distance above the cathode spot where the results of the more detailed one-dimensional near-cathode model discussed in [44] and the present two-dimensional model are both valid. This is the point where the quasi-neutral plasma begins to freely expand above the cathode region where the plasma is generated. In VAT the thrust is created in the cathode spot and therefore the thrust mechanism is not considered in this paper. The previous one-dimensional calculations further indicate that this plasma density corresponds to ion and electron temperatures of about $T_{\mathrm{i}}=0.5 \mathrm{eV}$ and $T_{\mathrm{e}}=2 \mathrm{eV}$, respectively, and are independent of the spot current. The ion temperature is a measurement of the random thermal velocity that is superimposed on the much larger directed velocity. The initial jet radius, $R_{0}$, is computed from $R_{0}=\left\{f_{\mathrm{i}} I_{0} /\left(\pi Z_{\mathrm{i}} e n_{0} V_{\mathrm{z} 0}\right)\right\}^{0.5}$, where $f_{\mathrm{i}}$ is the ion current fraction $\left(f_{\mathrm{i}}=0.1\right), I_{0}$ is the current per spot, $V_{z 0}=$ is the initial axial ion velocity $\left(1.3 \times 10^{4} \mathrm{~m} \mathrm{~s}^{-1}\right.$ in the case of $\mathrm{Ti}$ [16]), $n_{0}$ is the initial plasma density and $Z_{\mathrm{i}}$ is the ion mean charge. The region between the cathode surface and the starting plane in the present model includes the total potential cathode fall. This is the net voltage drop between the contact surface and the beginning of the freely expanding plasma region. When the total arc voltage is calculated in the present model, the cathode fall value is added to the plasma voltage so that the total arc voltage drop consists of the cathode fall and the plasma voltage. We define the free plasma boundary as the surface where the plasma jet velocity has only a tangential component, i.e. the normal component, $V_{\mathrm{n}}$, is zero. The plasma density at the free boundary of the plasma jet is $n=0$, and normal current density is $j_{\mathrm{n}}=0[36,37]$.

Thruster performance characteristics such as the thrust and specific impulse can be readily estimated based on the boundary conditions at the cathode side. In the case of a $100 \mathrm{~A}$ titanium arc the specific impulse is about $1300 \mathrm{~s}$ and the thrust is about $0.02 \mathrm{~N}$. 


\section{Magnetically enhanced co-axial vacuum arc thruster}

\subsection{Plasma plume in a magnetic field}

In this section we present results of the calculation of the plasma plume exhaust from the VAT based on the plasma flow model described above. Since the thrust is generated in the cathode spot, the plasma plume simply corresponds to the vacuum arc plasma jet emanating from the cathode spot. Specifically an effect of the magnetic field was studied. The geometry considered is shown in figure 2 . Since we are interested in plasma plume expansion at large distances from the cathode surface (several centimetres) it is necessary to take into account the magnetic field variation in the axial direction (far from the magnetic coil):

$$
\frac{B(z)}{B_{0}}=\frac{1}{\left[1+\left(z / R_{\mathrm{c}}\right)^{2}\right]^{3 / 2}} .
$$

This simple formula was obtained from the Biot-Savart law by integration over a circular current loop, where $B_{0}$ is the magnetic field at the cathode $(z=0)$ and $R_{\mathrm{c}}$ is the coil radius. It should be noted that the plasma detachment from divergent magnetic field is an important issue especially if the plasma acceleration coincides with the divergent magnetic field region [45]. In this model the radial component of the divergent magnetic field was neglected since plasma acceleration in the VAT occurs in the cathode spot which is a very small region attached to the cathode. In addition, in this model, we are investigating the plasma focusing by a magnetic field at relatively small distances from the plasma generation region. However, the far-field plume may be affected by the divergent magnetic field, and this problem requires special consideration going beyond the scope of this paper.

The calculated plasma density distribution in the plasma plume exhaust from the magnetically enhanced co-axial vacuum arc thruster (MVAT) is shown in figure 3. One can see that the magnetic field generally reduces plasma expansion. However, this effect takes place mostly near the plasma generation region (where the magnetic field is strong) and decreases in the axial direction. In order to enhance the focusing effect of an axial magnetic field, the cathode should be placed inside the solenoid (see figure 2). However, in this case the arc voltage can increase due to a decrease in conductivity across the magnetic field in the inter-electrode gap. The effect of the voltage rise will be considered in the next section.

In order to validate the model we performed a detailed comparison between the model predictions and the experimental results. The available experimental data contain the radial and axial distribution of the ion current density (experimental details can be found in a recent paper [12]).

The ion current density radial distribution is shown in figure 4 for several axial positions. Two cases are compared, with no magnetic field and with an axial magnetic field $\left(B_{0}=0.5 \mathrm{~T}\right)$. The plasma spreads significantly at large distances from the thruster and the plasma plume develops a half angle of about $45^{\circ}$. One can see that the magnetic field leads to plasma focusing and as a result the ion current density along the axis $(r=0)$ is high. It can be seen that the experimental and calculated distributions generally agree well and show similar trends.
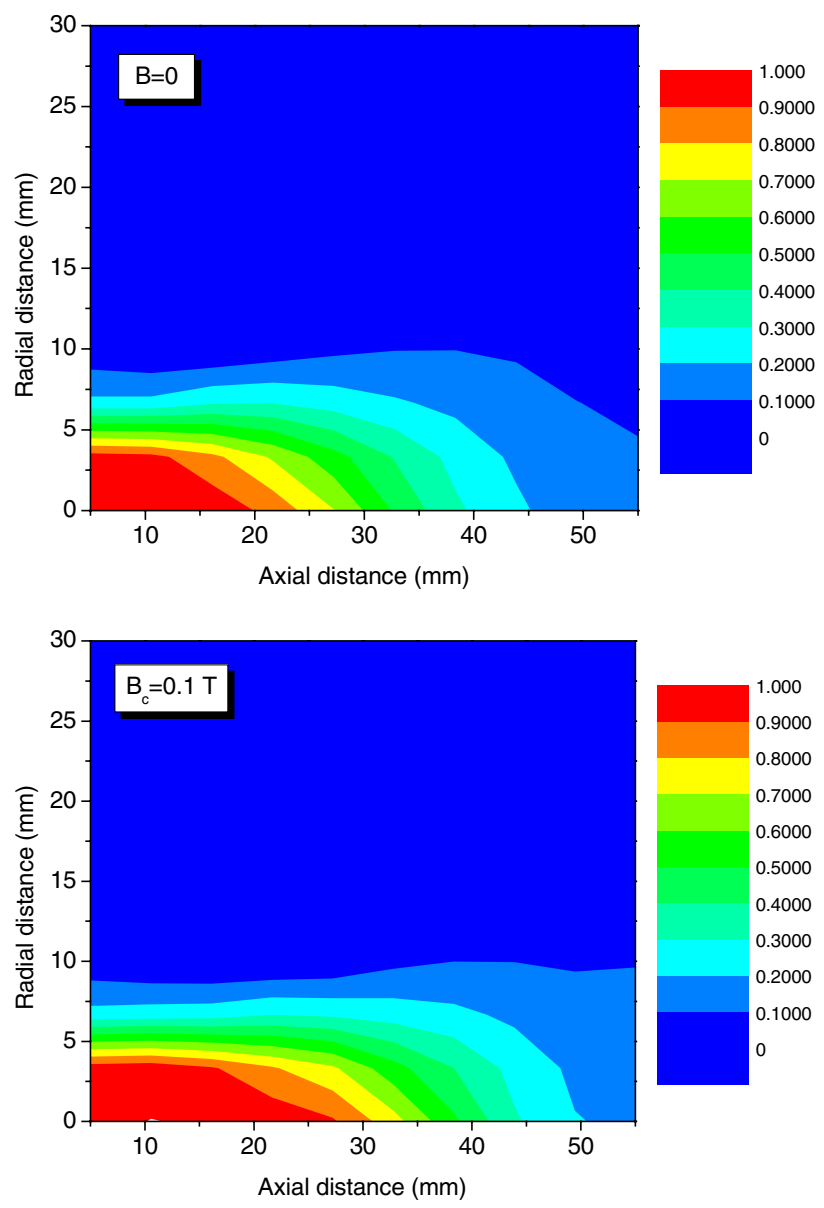

Figure 3. Comparison of the plasma density distribution with and without a magnetic field. The arc current is $200 \mathrm{~A}$. The magnetic field, $B_{0}$, is about $0.1 \mathrm{~T}$ at the cathode surface.

\subsection{Arc voltage}

In the previous section we considered the VAT configuration in which the solenoid was place flush with the cathode surface. It was suggested that a possible way of enhancing the effect of the magnetic field is to place the cathode inside the solenoid. Similarly, due to cathode erosion and therefore cathode recession after a long time of operation of the VAT, the cathode surface will be shifted inside the anode tube (and solenoid). However, in this case the arc voltage can increase due to a decrease in conductivity across the magnetic field in the inter-electrode gap [38]. Therefore it is important to understand how the cathode placement inside the solenoid will affect the arc voltage.

The potential distribution in the inter-electrode gap can be calculated from the current conservation equation (equation (4)), which can be written in the component form as follows,

$$
\frac{\partial j_{r}}{\partial r}+\frac{\partial j_{z}}{\partial z}+\frac{j_{r}}{r}=0
$$

where $j_{r}$ and $j_{z}$ are the radial and axial components of the current density, respectively, and are determined from equation (2). The numerical analysis is similar to that developed previously [36-40]. We use the implicit 

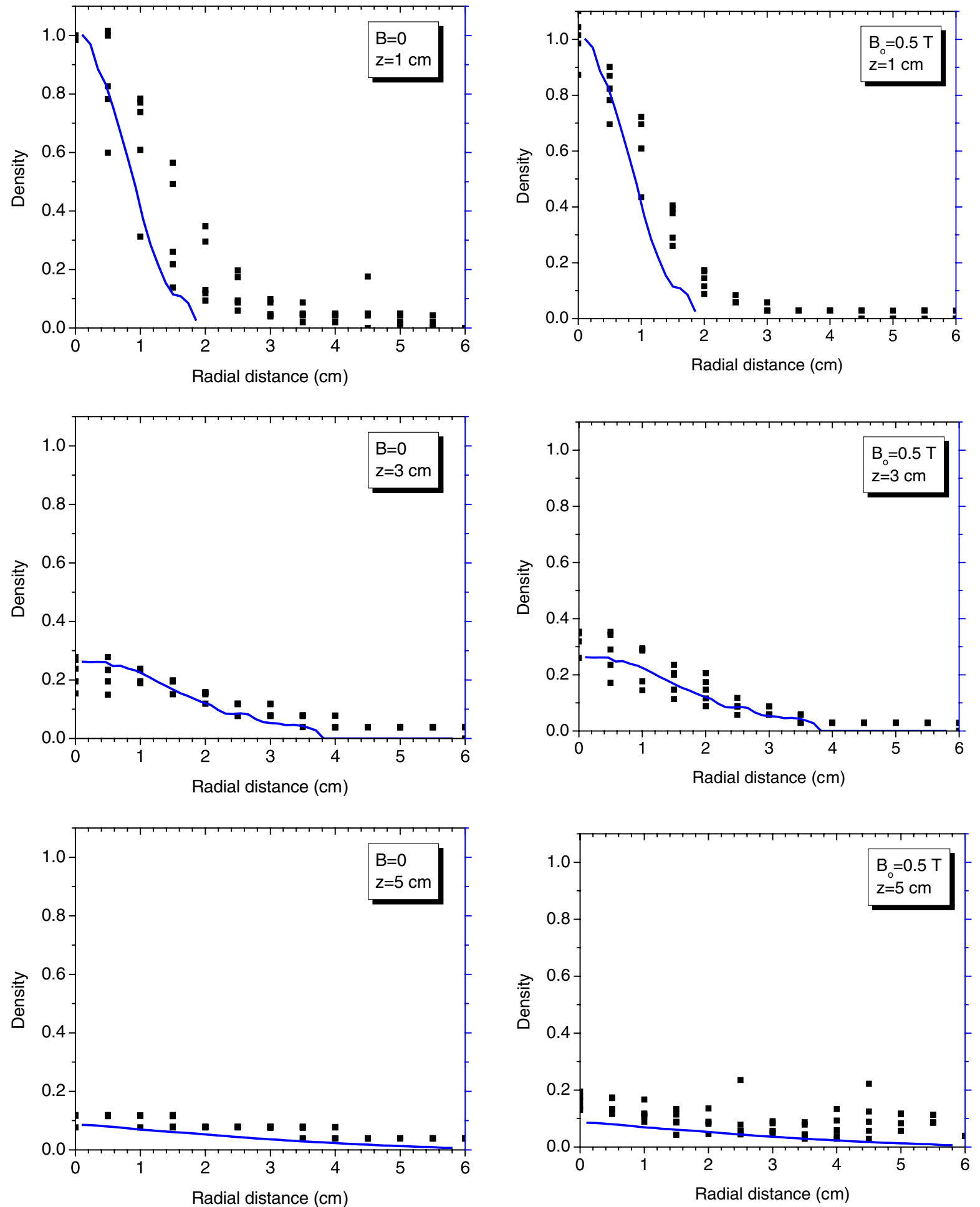

Figure 4. Ion current density distribution with and without axial magnetic field. Comparison with experimental results (experimental data: चם). Magnetic field near the cathode, $B_{0}=0.5 \mathrm{~T}$. Experimental data were taken from [12].

two-layer method to solve the system of equations (1)-(4). These equations are approximated by a two-layer, six-point scheme. An iterative procedure for finding the plasma density, velocity and potential distribution self-consistently is employed. The equation for potential (equation (6)) is solved numerically by iteration using the successive over-relaxation procedure.
The calculated potential distribution in the inter-electrode gap is shown in figure 5 for $B=0$ and arc current of about $200 \mathrm{~A}$. The inter-electrode distance (in the radial direction) is about $1 \mathrm{~mm}$. One can see that the potential drop in the quasineutral plasma in the absence of the magnetic field is about $1 \mathrm{~V}$.

Application of an axial magnetic field leads to an arc voltage increase as shown in figure 6 . It can be seen that a 


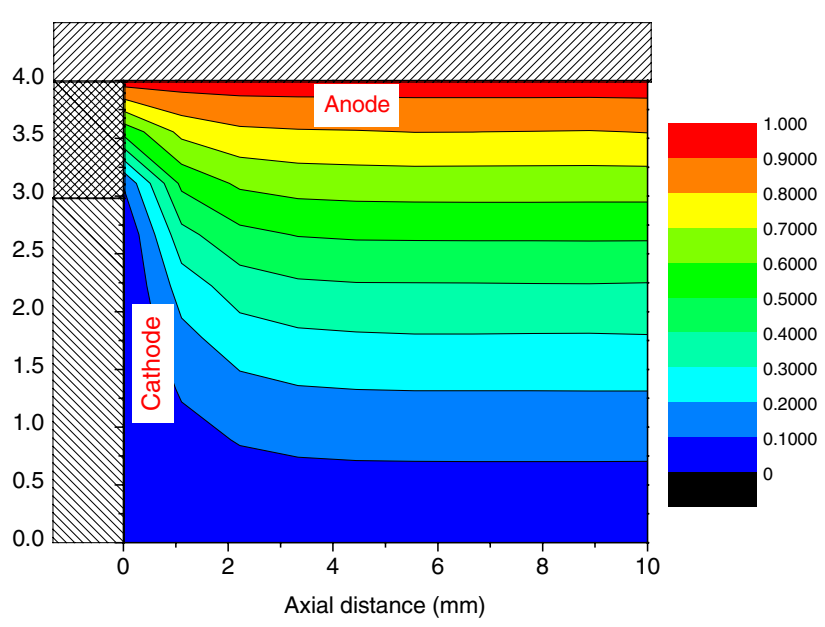

Figure 5. Potential distribution (in volts) in the vacuum arc inter-electrode gap of MVAT. $B=0$. The anode length is $10 \mathrm{~mm}$. Arc current is $200 \mathrm{~A}$. The cathode and anode voltage drops are not included.

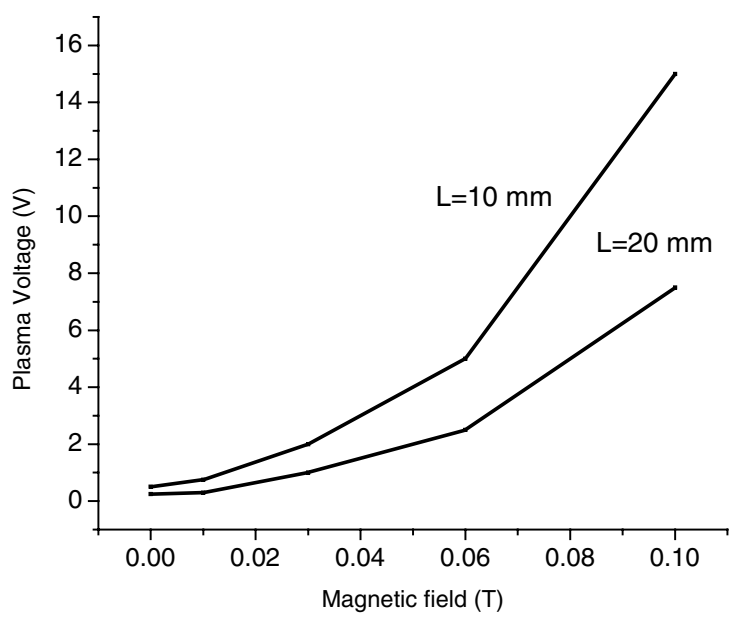

Figure 6. Vacuum arc voltage versus magnetic field with the anode length, $L$, as a parameter. The cathode and anode voltage drops are not included.

longer anode (cathode inside the solenoid) leads to a lower arc voltage. This effect can be explained by considering that the longer anode (and therefore collecting area) leads to a smaller current density and consequently to a smaller arc voltage. On the other hand plasma losses to the wall increase, as will be shown in the next section. Therefore one can conclude that optimization of the inter-electrode gap geometry (mainly the anode length) can be suggested to keep the arc voltage low while providing strong plasma collimation.

\section{Modelling of the ring electrode vacuum arc thruster}

In this section we present results of the simulation of a new VAT configuration. It was recently shown that a very effective feed mechanism is possible if the walled tube is used as a cathode as shown schematically in figure 7 [46]. Cathode spots are attached at the cathode-insulator interface, leading primarily to cathode edge erosion near that interface. By pushing the cathode towards the insulator (see figure 7), one can provide a long time operation of the thruster. A more detailed description of this thruster configuration can be found in [46].

However, in this case the thrust vector is not always directed along the axis of symmetry since the direction of the cathodic plasma jet can be normal to the cathode surface. The plasma generated by the single cathodic jets fills the cavity, creating a strong pressure gradient downstream. Therefore, thrust is generated by creation pressure gradient from the tube, i.e. VAT operates as an electrothermal thruster. This may lead to a lower exit velocity (specific impulse). In order to provide efficient thrust generation in this geometry and to preserve high ion velocity, a magnetic field can be used.

To this end a magnetically enhanced variant of the ring electrode VAT was developed and tested. In this section we present an analysis of the plasma flow inside the tube with the aim of explaining the experimentally observed non-monotonic behaviour of the ion current density with an axial magnetic field. The calculations are based on the model described in the section 3 .

Firstly, let us consider the cathodic plasma jet propagation across the magnetic field inside the thruster tube. The plasma jet expansion from the cathode spot across the magnetic field was calculated. The velocity distribution is shown in figure 8 with the magnetic field as a parameter. One can see that the plasma is decelerated in the course of flow across the magnetic field. The velocity decreases by a factor of 2 in the case of a magnetic field of about $0.1 \mathrm{~T}$. This effect can be explained by taking account electron magnetization and plasma quasineutrality conditions. On the other hand, the magnetic field collimates the plasma flow in the direction parallel to the field lines. The calculated ion flux $\left(n V_{\mathrm{z}}\right)$ distribution inside the tube (insulator and anode, see figure 7) is shown in figure 9 in the case of the plasma flow along the magnetic field. It can be seen that the axial magnetic field significantly affects the plasma flow and the ion flux at the thruster exit plane. On the other hand, plasma jet expansion leads to plasma deposition on the channel walls. The magnetic field decreases the plasma expansion as shown in figure 9 and leads to smaller plasma losses and a higher flux at the thruster exit plane.

Generally, two limited cases are possible with plasma jet propagation across the magnetic field in the cathode spot vicinity (and therefore a decrease of the plasma velocity as shown in figure 8) and plasma jet propagation along the magnetic field as shown schematically in figure 7 . These cases are determined by the cathodic jet formation in the cathode spot. The calculated ion current density at the thruster exit plane for two different jets, corresponding to two different cases, are shown in figure 10. One case corresponds to a cathodic jet initially directed along the magnetic field, and another case corresponds to the cathodic jet originating from the cathode in the direction transverse to the magnetic field. One can see that the ion current density increases with increasing magnetic field due to the plasma collimation effect; it reaches a maximum and then decreases due to the decrease in plasma velocity. On the other hand, the ion current density in the plasma jet propagating along the magnetic field experiences monotonic growth with the magnetic field and then saturates at a high magnetic field. For comparison the experimental data are shown (see $[12,46]$ for more details). It can be seen that the model prediction about the non-monotonic behaviour of the 

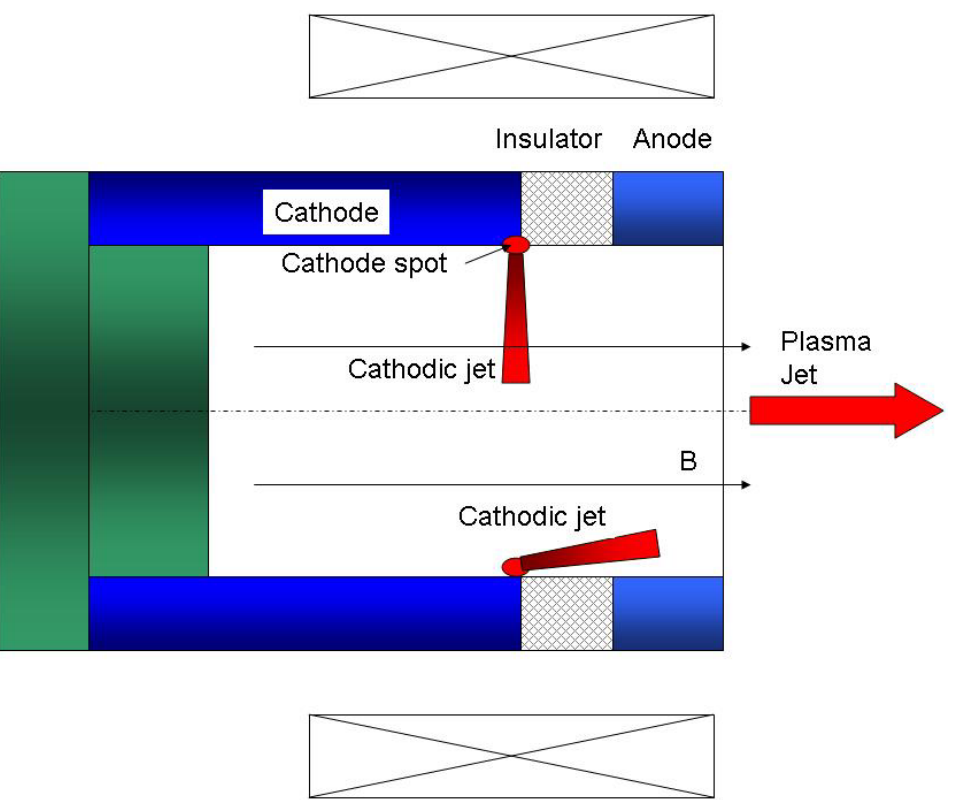

Figure 7. Feed mechanism and schematic representation of plasma collimation.

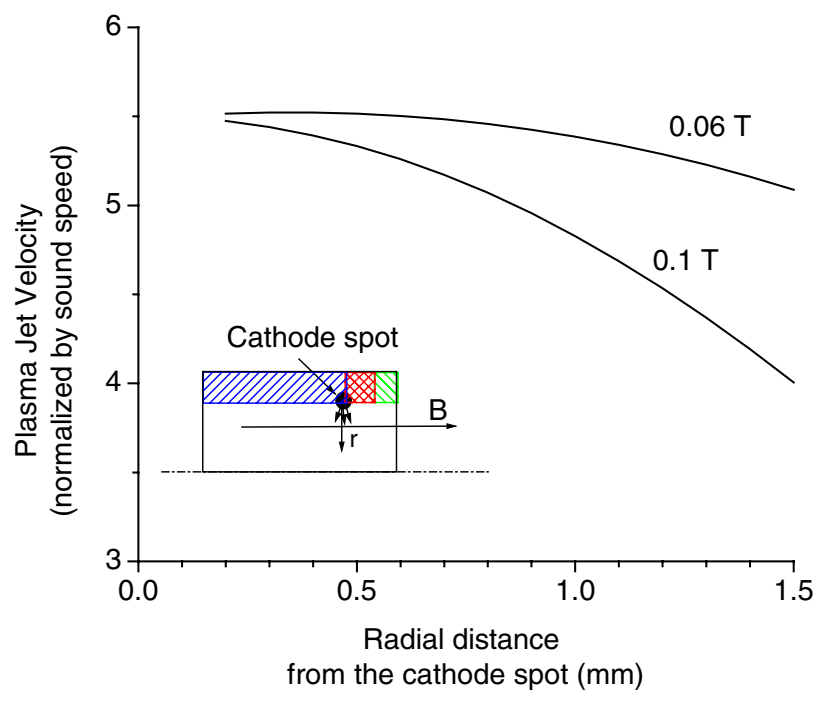

Figure 8. Calculated plasma jet velocity distribution in the radial direction (from the cathode spot) with a magnetic field as a parameter for the VAT configuration with a ring anode.

exhaust ion current density with magnetic field is in agreement with experimental trends. According to the model prediction the non-monotonic dependence of the ion current density on the applied axial magnetic field is seen in the case of the initial cathodic jet formation perpendicular to the magnetic field.

\section{Concluding remarks}

In this paper a hydrodynamic model of the MVAT and its plume was developed. A unified approach was used to study two VAT variants, namely a co-axial VAT and a ring anode VAT. The model of the vacuum arc plasma expansion was modified to account for the peculiarities of the VAT, such as the magnetic field weakening in the plume in the case of the co-axial
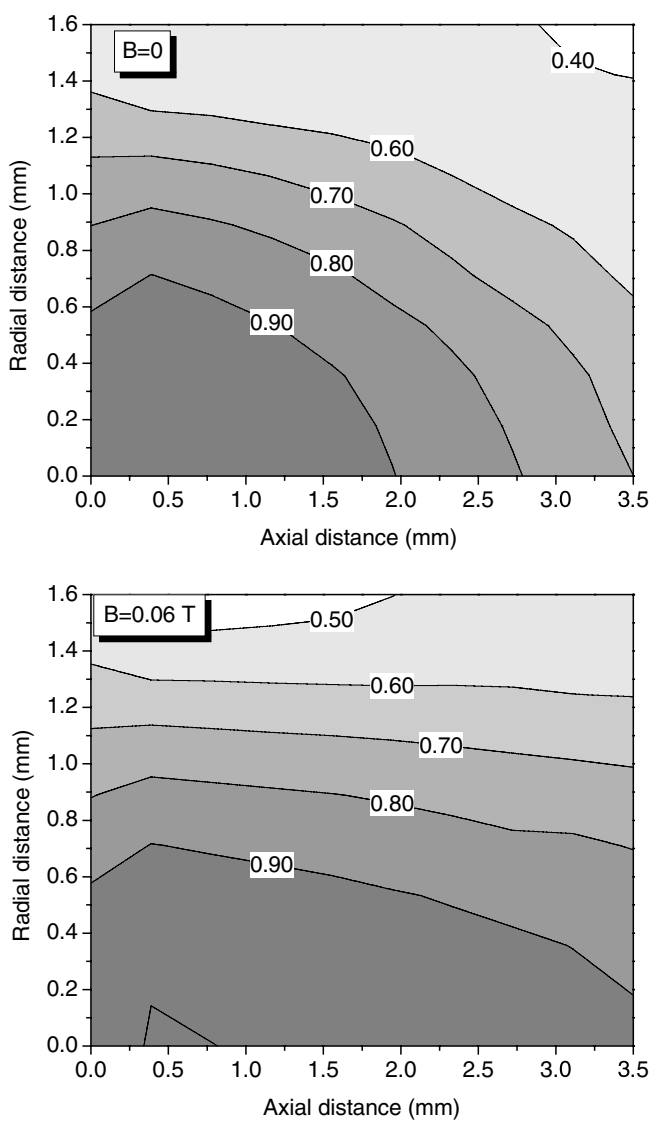

Figure 9. Ion flux distribution (ion flux values are normalized by initial ion flux at the cathode-insulator plane) inside the tube between the cathode plane and the exit.

configuration and cathodic jet generation across the magnetic field in the case of the ring anode configuration. According to the model prediction a magnetic field can be chosen by trading between two conflicting tendencies of plume expansion 


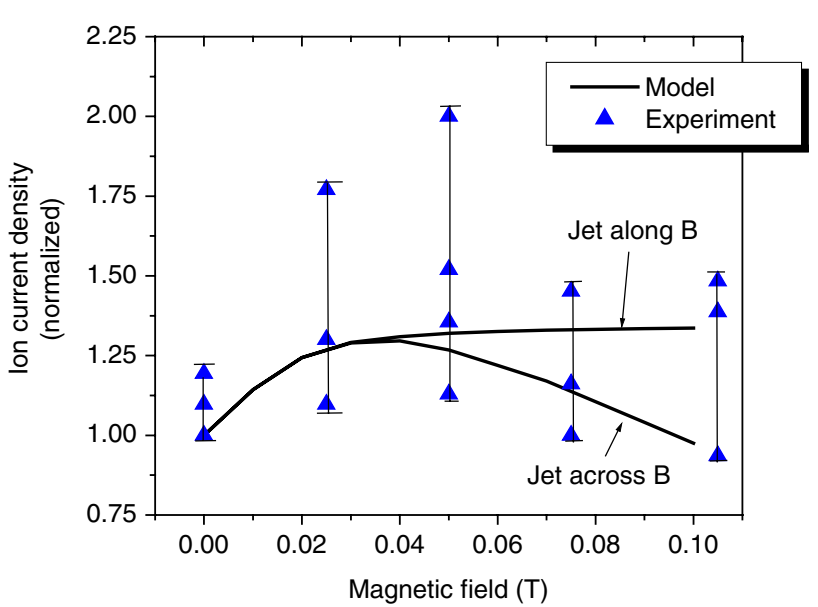

Figure 10. Ion current density (exit plane) dependence on the magnetic field inside the tube. Comparison of the model predictions and experimental results [46].

reduction with magnetic field increase on the one hand and arc voltage (and therefore power) increase with magnetic field increase on the other hand. Limiting plume expansion and keeping the arc power low are specifically important for spacecraft propulsion applications. Various model predictions, such as plume expansion and ion current density variation with applied magnetic field, were validated against experimental data, and generally good agreement was found. In the case of a ring anode VAT it was found that the magnetic field leads to plasma jet deceleration under typical conditions. This effect explains the non-monotonic dependence of the ion current density on the magnetic field observed experimentally.

\section{Acknowledgment}

This work was funded in part by NASA SBIR Contract No NNC04CA19C.

\section{References}

[1] Gilmour A S and Lockwood D L 1972 Proc. IEEE 60 977-91

[2] Dorodnov A M 1978 Sov. Phys. Technol. Phys. 23 1058-64

[3] Dethlefsen R 1968 AIAA J. 6 1197-9

[4] Qi N, Gensler S, Prasad R R, Krishnan M, Visir A and Brown I G 1998 34th Joint Propulsion Conf., July 1998 AIAA-Paper-98-3663

[5] Qi N, Schein J, Binder R, Krishnan M, Anders A and Polk J 2001 37th Joint Propulsion Conf. (Salt Lake City, UT, 8-11 July 2001) AIAA-2001-3793

[6] Schein J, Anders A, Binder R, Krishnan M, Polk J E, Qi N and Ziemer J 2002 Rev. Sci. Instrum. 72 925-7

[7] Schein J, Krishnan M, Ziemer J and Polk J 2002 Nanotech Conf. AIAA paper 2002-5716

[8] Schein J, Gerhan A, Rysanek F and Krishnan M 2003 Vacuum arc thruster for cubesat propulsion IEPC-0276: 28th IEPC

[9] Gilmour A S 1966 AIAA 5th Electric Propulsion Conf. (San Diego, CA, March 1966) AIAA paper 66-202
[10] Gilmour A S, Clark R J and Veron H 1967 AIAA Electric Propulsion and Plasmadynamics Conf. (Colorado Springs, CO, September 1967) AIAA paper 67-737

[11] Anders A, Anders S and Brown I G 1995 Plasma Sources Sci. Technol. 4 1-12

[12] Au M, Schein J, Gerhan A, Wilson K, Tang B and Krishnan M 2004 40th AIAA Joint Propulsion Conf. (Fort Lauderdale, FL, July 2004) AIAA paper 2004-3618

[13] Djakov B E and Holmes R 1974 J. Phys. D: Appl. Phys. 7569

[14] Boxman R L, Martin P J and Sanders D M (ed) 1995 Vacuum Arc Science and Technology (New York: Noyes)

[15] Juttner B 2001 J. Phys. D: Appl. Phys. 34 R103

[16] Kutzner J and Miller H C 1992 J. Phys. D: Appl. Phys. 25 686-93

[17] Plyutto A A, Ryzhkov V M and Kapin A T 1965 Sov. Phys. JETP 20328

[18] Davis W D and Miller H C 1969 J. Appl. Phys. 402212

[19] Lafferty J M (ed) 1980 Vacuum Arcs-Theory and Applications (New York: Wiley)

[20] Beilis I I 2001 IEEE Trans. Plasma Sci. 29 657-70

[21] Aksenov I I, Padalka V G and Khoroshikh V M 1979 Sov. J. Plasma Phys. 5 341-4

[22] Cohen Y, Boxman R L and Goldsmith S 1989 IEEE Trans. Plasma Sci. 17 713-6

[23] Brown I G 1994 Rev. Sci. Instrum. 653061

[24] Oks E M, Brown I G, Dickinson M R, MacGill R A, Emig H, Spadtke P and Wolf B H 1995 Appl. Phys. Lett. 67 200-2

[25] Yushkov G, Anders A, Oks E and Brown I G 2000 J. Appl. Phys. 885618

[26] Aksenov I I and Khoroshikh V M 1998 Proc. 18th Int. Symp. Disch. Elect. Insul. Vac. (ISDEIV) (Eindhoven, 1998) p 570

[27] Anders A and Yushkov G Y 2002 J. Appl. Phys. 914824

[28] Lyubimov G A 1977 Sov. Phys.-Tech. Phys. 22173

[29] Wieckert C 1987 Phys. Fluids 301810

[30] Beilis I I, Zektser M P and Lyubimov G A 1988 Sov. Phys.Tech. Phys. 33 1132-7

[31] Beilis I I and Zektser M P 1991 High Temp. 29 501-4

[32] Robertson R 1938 Phys. Rev. 53578

[33] Compton K 1930 Phys. Rev. 36706

[34] Tonks L 1934 Phys. Rev. 46278

[35] Kobel E 1930 Phys. Rev. 361636

[36] Keidar M, Beilis I I, Boxman R L and Goldsmith S 1996 J. Phys. D: Appl. Phys. 29 1973-83

[37] Beilis I, Keidar M, Boxman R L and Goldsmith S 1998 J. Appl. Phys. 83 709-17

[38] Keidar M, Beilis I, Boxman R L and Goldsmith S 1997 IEEE Trans. Plasma Sci. 25 580-5

[39] Beilis I I, Keidar M, Boxman R L and Goldsmith S 1999 IEEE Trans. Plasma Sci. 27 872-6

[40] Keidar M and Schulman M B 2001 IEEE Trans. Plasma Sci. 29 684-9

[41] Keidar M, Beilis I I and Brown I G 1999 J. Appl. Phys. 84 5956-60

[42] Keidar M, Brown I G and Beilis I I 2000 Rev. Sci. Instrum. 71 698-700

[43] Beilis I I and Keidar M 2002 Proc. 20th Int. Symp. Disch. Elect. Insul. Vac. (Tours, 2002) pp 535-8

[44] Beilis I I 1995 Handbook of Vacuum Arc Science and Technology ed R L Boxman et al (New York: Noyes)

[45] Arefiev A V and Breizman B N 2004 Phys. Plasmas 11 2942-9

[46] Wilson K, Schein J, Gerhan A, Au M, Krishnan M 2004 40th AIAA Joint Propulsion Conf. (Fort Lauderdale FL, July 2004) AIAA paper AIAA 2004-3617 